\title{
PHOTOGRAMMETRY FOR MAPPING UNDERGROUND UTILITY LINES WITH GROUND PENETRATING RADAR IN URBAN AREAS
}

\author{
N.E. Cazzaniga ${ }^{\mathrm{a}}$, D. Pagliari ${ }^{\mathrm{a}}$, L. Pinto ${ }^{\mathrm{a}^{*}}$ \\ ${ }^{a}$ DIIAR - Surveying, Geodesy and Geomatics Section, Politecnico di Milano, Piazza Leonardo da Vinci 32, Milan - Italy \\ (noemi.cazzaniga, livio.pinto)@polimi.it,diana.pagliari@mail.polimi.it
}

\author{
Commission I, WG I/5
}

KEY WORDS: Automation, Orientation, Navigation, Urban, Georeferencing, Mobile, Sequences, Calibration

\begin{abstract}
:
Ground Penetrating Radar (GPR) is an active instrument often used to detect underground utility locations up to a few meters. To perform a three-dimensional reconstruction of position and geometry of the surveyed features, the accuracy of GPR position data has to be in the order of $20-30 \mathrm{~cm}$. This requirement is easily attainable using a GNSS system in open sky conditions, while in urban areas signal leakage is frequent, leading to inadequate position accuracy or even positioning failure. Usually, in those cases, GPS/INS navigation systems are used, but they are quite an expensive solution. To determine the position of the GPR, another strategy could be utilizing a photogrammetric method that uses information extracted from a large scale map, often available for towns. In this paper, the characteristics of this procedure and some possible configurations of cameras are described. Results obtained from preliminary tests are hereby presented and discussed to demonstrate that the proposed methodology could achieve the required precision.
\end{abstract}

\section{INTRODUCTION}

\subsection{GPR georeferencing}

A problem of topical interest for Civil and Environmental Engineering is the lack of detailed knowledge about the location of underground utilities, such as pipes, ducts, cables, etc., that serve both civil and industrial users. Therefore, a company planning the installation of new facilities needs an adhoc underground survey. One of the most used methods to detect underground network utility positions and geometry up to a few meters is the Ground-Penetrating Radar (GPR), suitably georeferenced, which allows variable accuracies, depending on specific soil type and on the material of the utilities themselves. The GPR is an active instrument that transmits electromagnetic radiation and detects the reflected signals from subsurface structures to picture them. It is typically mounted on a trailer pulled by hand or by a vehicle. The maximum allowable operating speed is around $15-20 \mathrm{~km} / \mathrm{h}$, however the daily productivity is usually around $3 \mathrm{~km}$. In order to provide a three-dimensional representation of the underground surveyed objects, this instrument has to be planimetrically georeferenced (not necessarily in real time) with an accuracy of about $20-30 \mathrm{~cm}$. This requirement is easily attainable using GNSS system in open sky conditions. Contrariwise, in urban areas, the presence of obstacles, such as high buildings, trees, tunnels etc., causes frequent signal leakage and multipath interference, often leading to inadequate position accuracy when signal quality is poor or even to no positioning at all if the number of visible satellites is less than four. Usually, to overcome this limit, an INS/GPS (GPS-aided Inertial Navigation System) is adopted, often combined with a DMI (Distance Measurement Instrument). In many cases this approach can overcome the drawback of the GPS-alone solution, especially in a tightly-coupled fashion. As known, INS/GPS accuracy decreases quite quickly over time, when less than four satellites are visible. The most recent systems allow a positioning error in the order of $10 \mathrm{~cm}$ after a GPS outage of almost 1 minute. Thus, this solution does not seem to be suitable for our aims, since the low speed of the GPR carrier can cause a very long period of GPS leakage and consequently unacceptable large drift errors. Moreover, inertial aided navigation is still rather expensive even if remarkable improvements in the field of low cost inertial systems (MEMS) have been done. Another possibility is the use of robotic total stations with auto-tracking able to auto-point a target placed on the GPR carrier. This solution is often applied to GPR surveying, especially in closed environments such as warehouses. Some drawbacks of this approach are the need for more stations and the frequent interruption of the line of sight, leading to loss of prism lock due to car traffic and other obstacles nearby the roadway.

\subsection{Photogrammetry as a navigation system}

Photogrammetry can be useful as a low cost navigation system that can overcome GPS signal leakage in urban areas. Automatic image sequence orientation to support an INS/GPS system to overcome GPS outages has already been proposed (Chaplin and Chapman, 2001; Tao et al, 1999; Roncella et al, 2005). Photogrammetry has been used to aid low cost INS/GPS, as presented in (Alshawa et al, 2009). Some examples of Mobile Mapping Systems (MMS) that use photogrammetry as a backup when navigation solution is not available are presented in (El-Sheimy et al, 1995; Hassan et al, 2007) too. In those cases, the position of the first and last frame, where the INS/GPS solution is available, are introduced in the bundle adjustment as observed parameters.

During GPS outages, a joined application of position and orientation data of a low-grade IMU (Inertial Measurement Unit) with Structure and Motion (S\&M) reconstruction is possible. (Forlani et al., 2005) demonstrated that a purely photogrammetric approach can be used to define the vehicle trajectory in case of long GPS outages and (Da Silva et al., 2003) developed a prototype of MMS in which, in order to keep the system cheaper, photogrammetry alone was used to supply 
GPS outages. In this case, GPS is used to constrain the perspective center of the extreme stereobases of an image pair. The major limit of this approach is the drift of the solution; however in this case the drift is a function of covered distance, and not of time as for INSs. Concerning the achievable accuracy, (Forlani et al., 2005) demonstrated that the projection centers of a sequence of front stereo image acquired on a mobile van over a path of $300 \mathrm{~m}$ can be determined with an error in the order of $1 \mathrm{~m}$.

However, this result can be improved by adding some additional constraints, e.g. considering some ground control points (GCP) of known coordinates. In urban areas, digital base maps are often available on a large scale. Many elements obtainable from them and visible on the acquired image can be used as GCPs to solve an inverse photogrammetric problem. Using digital map points to evaluate the external orientation of a mobile sensor was already proposed by (Crosilla and Visintini, 1998). In that case a solution was obtained by applying a forward Kalman filter: some points were extracted from a map and interpolated with spline functions to determine the dynamic model (steady-state equation of the filter). In that way it was possible to determine the external orientations by using a camera and an odometer. Instead, we propose to solve the photogrammetric problem with a bundle adjustment, by using some GCPs whose coordinates are obtained from a large scale map. In fact, the façades of the buildings located on the roadside can potentially provide a high number and a good distribution of tie points, allowing the utilization of matching algorithms as well.

The best configuration seems to be the one with the camera pointing rightwards in the direction of the building façade. This configuration ensures minimal image scaling and rotation thereby strengthening the solution and simplifying tie points extraction on the overlapping area.

A recent study (Barzaghi et al., 2009) demonstrated the potential of such a solution, performing some simulations in the city of Milan. The vehicle was supposed to be equipped with a GPS and two digital cameras pointing leftwards and rightwards respectively. The tie points were represented by a regular point grid on the building façade, with regular spacing of $4 \mathrm{~m}$. GCPs were identified at each building corner, considering that the images include the ground level at the bottom of the building block. Three different scenarios, with different focal lengths at different distances from the façade, have been simulated. The results suggest that the projection centers of the cameras can be georeferenced with precision below $10 \mathrm{~cm}$, if GCPs can be recognized on images at intervals not larger than 200-250 m.

Because this approach is quite demanding, particularly concerning the required time for post-processing and the tricky automation of some steps, we consider the photogrammetric strategy just as a backup solution whenever GPS positioning is not available with the desired accuracy. Both techniques are meant to collect data simultaneously, but not all gathered images are processed.

In this paper we present the first results of a study aimed to implement the described photogrammetric approach, aided by cartography. First of all, we describe the characteristics of the method and highlight the major tasks in real cases. We also analyze possible solutions, principally aiming towards a high level of automation for the procedures of GCPs extraction from maps and extraction of homologous points. We then compare some possible configurations of the onboard cameras in order to optimize their configuration. In the successive paragraphs we present results from some tests. The first trial consisted in acquiring images of the building façades by manually moving a digital camera. To prove the obtained accuracy we materialized a topographic network to accurately measure some check points. We describe a test with a vehicle too. The vehicle was equipped with cameras and a GPS receiver. The first survey was performed using a stop-and-go approach, manually controlling camera shooting. The GPS solution was used to check the results. A kinematic test was then performed in which the vehicle was equipped with two digital cameras and a GPS antenna. Finally, the results were discussed and some possible improvements presented.

\section{THE PROPOSED APPROACH}

The investigated method requires a digital camera and a GPS antenna mounted on a trailer carrying the GPR. The camera is pointed at the building façades on the right side with its axis approximately perpendicular to them so that one photogrammetric strip is acquired at each pass of the trail. To georeference the photogrammetric strip, the interior and exterior orientation parameters have to be determined. First of all, a camera calibration is performed in order to estimate the interior orientation of the camera. The interior orientation parameters so estimated can be considered constant over the survey (Grejner-Brzezinska, 1999). Using robust S\&M algorithms, homologous points can be extracted. The exterior orientation parameters change quickly as the vehicle moves: they can be reconstructed with a bundle adjustment procedure, constrained with GCP coordinates automatically extracted from a large scale digital map. Obviously, the position of the GPR antenna can be determined because the navigation systems (camera or GPS antenna) are rigidly attached on it. The position of the GPS antenna phase centre and the rigid transformation (rototranslation) from the camera system to a vehicle-fixed reference system (Figure 1) can be determined during a calibration stage (cfr. 3.1)

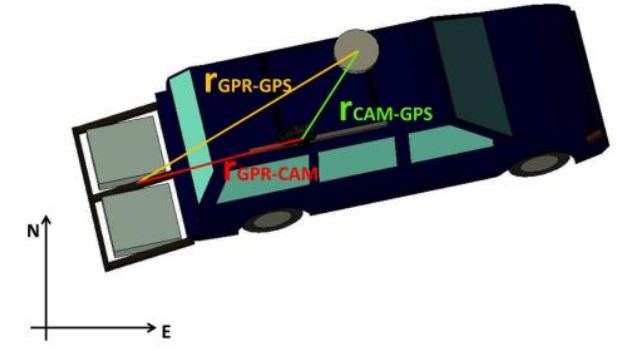

Figure 1 - Reference systems

\subsection{Automatic extraction of tie points}

Tie points may be tracked with S\&M techniques on a large number of images. The software used during the tests that will be present later on is EyeDEA, developed by University of Parma (Roncella et al., 2011a; Roncella et al., 2011b). It implements the SURF operator and the SURF feature descriptor (Bay et al., 2008), allowing to identify a large number of tie points and to manage a long image sequence.

The identification of tie points is more difficult in close range photogrammetry compared to aerial photogrammetry due to convergences, presence of occlusions, large scale variations and variable overlapping between the images. The integration of traditional photogrammetry and projective techniques allows combining the rigorous stochastic model of the traditional methods with the high level of automation and independence from a priori information of the latter. 
In order to speed up the photogrammetric orientation process the tie points need to be extracted using an interest operator. The urban environment presents a huge number of details that make it easier to well define interest points.

Because any interest operator will return a large number of matches, with erroneous correspondence within each set, a robust method has been implemented in EyeDEA. First of all, the constraint between two sets of coordinates is implemented through the fundamental matrix F. Because the epipolar constraint is not sufficient to discriminate the wrong matches if the two points are located on the epipolar line, the Trifocal tensor is then implemented to identify the erroneous correspondences in triplets of images. The RANSAC paradigm (Fischler and Bolles, 1981) is implements after each geometric control to achieve a higher percentage of inliers.

This algorithm is very useful in image analysis because it can deal with data containing a high percentage of gross errors, such as data extracted with interest operators.

When a long image sequence is processed, it is fundamental to optimize the distribution of tie points and reduce the amount of data while still preserving the multiplicity feature. In fact, if the number of tie points is too large, the photogrammetric block orientation will be difficult to manage. The point number reduction was performed by a Matlab ${ }^{\circledR}$ function. The decimation was carried out according to the criteria of homogeneous distribution throughout the block and high point multiplicity. In this way we basically had no influence on the final accuracy although we significantly increased the speed of the orientation solution.

From a large number of tests, it has also emerged that assuring a good distribution of the homologous points on the image is necessary to pre-process the strip with the Wallis filter (Wallis, 1976). This filter underlines local contrast preserving object details which allows to extract features even where the image texture is not so evident.

Subsequently, the whole sequence is oriented with a bundle block adjustment that provides the external orientation of the images (as well as the ground coordinates of the image tie points). By accounting for the transformation from camera system to vehicle system, the external orientation parameters can be converted in photogrammetrically-derived vehicle positions. Then, thanks to the rigid transformation defined during the calibration stage, both the GPS and the photogrammetric solution can be referred to the GPR antenna. Obviously, all sensors (GPR, camera and GPS) need to be synchronized in time.

\subsection{Automatic extraction of GCPs from urban maps}

In order to georeference the photogrammetric strip and reduce solution drift, an urban map of the surveyed area can be used to extract the coordinates of some significant points that may be exploited as GCPs. These points, such as building corners, perimeter fences, sidewalk borders etc., are only potentially visible on images because of the possible presence of obstacles like parked cars or trees.

We implemented an automated procedure with some scripts to make GCP extraction less time-consuming and avoid gross errors due to manual extraction. A script realized with ArcGIS Desktop ${ }^{\circledR}$ Model Builder application (ESRI) is used to extract the planimetric coordinates of all the vertices of the polyline that composes any building polygon. The points that effectively represent building corners are selected using a function created in Matlab®. This function verifies if an angle between two edges, defined by three consecutive points, is wider than a threshold value. If so, the point is recognized as a building corner (Figure 2).

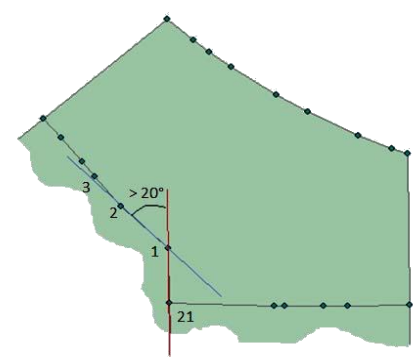

Figure 2. The criteria to identify building corners

The extracted GCP elevation is defined considering the altimetric coordinates of the nearest spot height. This is necessary because we are using a 2D numerical map in which the heights of only some spot points are available, the latter not necessarily being coincident with the building corners.

\section{INSTRUMENT CONFIGURATIONS}

The cameras used in the tests presented below are two Nikon D70 SLRs, with a lens-wide angle of $20 \mathrm{~mm}$. The camera frame is rectangular, composed of 3008 columns and 2000 lines of pixels. The pixel size is about 7.1 microns. To define their optimal configuration we performed a series of simulations.

We considered configurations with nadiral and slightly convergent cameras spaced apart to ensure overlapping between frames of $60 \%$ and $75 \%$ respectively. The achievable perspective center accuracy did not show substantial differences. In any case, the accuracy are about one order of magnitude lower than the required tolerance. However, using two slightly convergent cameras would guarantee to have less frequent shots thereby preserving a sufficient overlapping between subsequent frames at the same time.

For each test presented below we calibrated the cameras to define the interior orientation parameters with a point cloud calibration using the dedicated procedure of the commercial software Photomodeler®.

\subsection{Geometric calibration of the instruments}

The proposed method requires the installation of all position devices used (cameras and GPS antennas) on the same vehicle. To compare different instrument solutions, and in view of an integration of their data, it is necessary to compute their relative positions. The $3 \mathrm{D}$-vectors that connect each camera with the GPS antenna are defined during geometric calibration. Both the camera set-up and the antenna position are invariant in a vehicle-fixed reference system, so it is possible to determinate the vectors by taking a series of images of some targets of known coordinates. The calibration polygon has to be an artifact characterized by a stable geometry and dimension similar to those of the buildings that will be present in the acquired frames. It also needs to be easily accessible with the vehicle and characterized by high color contrast and sharp and evident details. In our case we used the façade of a building of the Politecnico di Milano, which fulfills all these conditions, after measuring on it six well-identifiable points.

A series of simulations was performed with the scientific software Calge (developed at Politecnico di Milano) with the aim to define the most favorable acquisition geometry for the calibration step. We suppose to know the coordinates of 48 points, regularly distributed along 3 rows, at the height of 1,5 
and $9 \mathrm{~m}$ from the ground. Four different configurations have been simulated: firstly, we considered three convergent acquisitions $15 \mathrm{~m}$ away from the building façade. For the second simulation we inserted two more acquisitions interposed between the three mentioned above. The third and the fourth simulations were realized respectively with 5 and 9 nadiral acquisition. Also, in these cases, the distance from the building façade was about $15 \mathrm{~m}$. From our tests, it has emerged that the preferable configuration is the one with 5 convergent acquisitions, with a distance of $5 \mathrm{~m}$ between the subsequent station points.

Afterwards, we took a series of photos of the calibration polygon with the vehicle (already equipped with all the instruments that we would use during the survey) while we were acquiring the GPS coordinates of the station points. We collimated a series of well distributed tie points (using the six points as control points) and solved the photogrammetric problem with a bundle-adjustment.

\section{TESTS}

\subsection{Some preliminary tests}

We initially performed a static test, with the purpose to evaluate the precision achievable with the proposed method. The test was executed without the vehicle and the camera, installed on a tripod, was manually moved along a path similar to the one that the GPR might follow during its operational phase.

We acquired a series of images along viale Romagna, a street in Milan (Italy), already considered in the simulation performed by (Barzaghi et al., 2009). The survey was organized with 97 station points, for a total length of about $300 \mathrm{~m}$. Each acquisition was spaced $3 \mathrm{~m}$ from the other to guarantee a standard overlapping $(60 \%)$ between one frame and the following one. The image was taken at $10 \mathrm{~m}$ from the buildings, keeping the camera parallel to the façades. This allowed to recover part of the sidewalk, the ground floor and the first floor of the buildings, with the aim to recreate the conditions presented in the simulations.

On the images taken, usually shooting building façades, it was possible to identify 607 homologous points. We performed the bundle-adjustment of the photogrammetric block using some GCPs extracted from a base map of Milan city on a 1:1000 scale. Beside the coordinates of GCP points that referred to elements like building corners, located at the ground floor of the buildings themselves, we also included some altitude points, with the aim to avoid singular or badly conditioned configurations of the photogrammetric block.

The standard deviation on the camera projection centers is in the order of $10 \mathrm{~cm}$, in agreement with our requirements.

In order to verify the bundle block precision, a topographic network was materialized and measured. The traverse survey involved 10 station points, spaced about $30 \mathrm{~m}$ from each other, in order to cover the same area of the photogrammetric block. We collimated 24 points on the building façades which were also photogrammetricaly measured on the acquired image.

\begin{tabular}{|c|c|c|c|}
\hline & $\mathrm{E}(\mathrm{m})$ & $\mathrm{N}(\mathrm{m})$ & $\mathrm{h}(\mathrm{m})$ \\
\hline St.dev & 0.033 & 0.058 & 0.053 \\
\hline Min & -0.066 & -0.075 & -0.095 \\
\hline Max & 0.069 & 0.143 & 0.081 \\
\hline
\end{tabular}

Table 3. Residuals of the photogrammetric coordinates with respect to those topographically surveyed
The residual coordinates of the photogrammetric points with respect to the topographic coordinates are reported in Table 3. The data show that accuracies are sub-decimetric, in line with those required.

In a second test, the vehicle was equipped with a single camera, remotely controlled by a laptop, and a GPS receiver. The camera was considered as the unique positioning instrument, while the GPS antenna allowed for a solution of good and controllable accuracy yielding reference positions. Then, we chose a street that could ensure good GNSS signal reception. This was to guarantee the highest possible number of GPS coordinates. We chose via Camillo Golgi in Milan. The street is characterized by building façades having a good texture.

During this test, we performed a stop and go survey with the vehicle which was stopped each time the camera took a picture. This choice was made in order to avoid introducing errors while synchronizing of the camera with the GPS. We performed our test in a $150 \mathrm{~m}$ long section and the camera was orthogonal to the building facades. The photogrammetric block consisted in 44 frames and roughly 40 homologous points were manually extracted from each of them.

To quantify the precision of the solution obtained with the photogrammetric method we considered the residuals between the camera coordinates estimated via the new approach and the GPS ones. They were compared to the tolerance required to georeference the GPR and demonstrated that the results had an even better accuracy than the required one. The standard deviation of the residuals was in the order of $10 \mathrm{~cm}$.

\subsection{The kinematic test}

We performed a kinematic survey too, with the purpose of simulating the operating conditions. We equipped the vehicle (Figure 4) with two cameras rigidly fixed to a bar mounted on the vehicle carrier and two GPS antennas. The data received from the second GPS antenna were not used in the solution hereby presented: they were acquired though we expect to integrate them within the photogrammetric block.

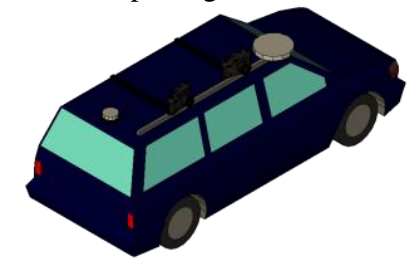

Figure 4. The vehicle equipped with the instruments used during the kinematic survey

The two cameras were remotely controlled with a laptop and shooted almost synchronously (with a difference of just few milliseconds). For each camera, the image acquisition times were stored in PC time in an ASCII file thanks to a remote control software written on purpose. The synchronization between the two cameras and the GPS time has been performed by software as well. The GPS time was stored together with the PC time and then the transformation between the two time scales was estimated via least-squares interpolation.

The survey was performed in the same street of the stop and go test, via Camillo Golgi, Milan, for a total length of about $350 \mathrm{~m}$. From this last test we extracted the homologous point using the EyeDEA software presented so far. The software was able to extract 308,658 image points. Such a high number of points would make it difficult to manage the photogrammetric block and would require too long of a computational time. Therefore points were filtered according to the previously presented 
criteria. The image points actually used for the bundleadjustment procedure were then reduced to 28,485 , corresponding to 7822 tie points. The number of extracted seed points varied as a function of the façade texture and of the presence of some kind of obstacles. For example, in the image shown in Figure 5, the software could not extract tie points in correspondence with the asphalt, the sky, or the parked vehicles. The points extracted in correspondence with the leaves have been manually removed because we did not consider them as reliable ones because they could move between subsequent acquisitions.

To georeference the photogrammetric block we used 11 GCPs extracted from the urban map (with a planimetric accuracy of $20 \mathrm{~cm}$ and an altitude accuracy of $30 \mathrm{~cm}$ ), so that the reference system would be the cartographic one. Those points were located in correspondence with walls and building corners.

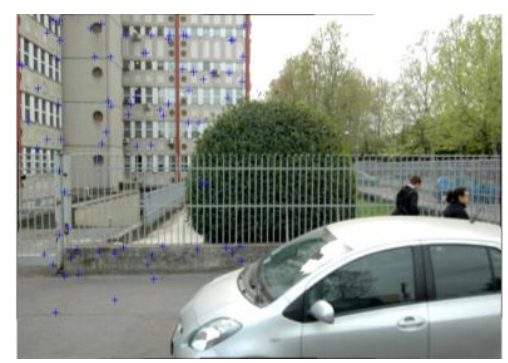

Figure 5. Problems in automatic tie points extraction in presence of obstacles

The image pairs were acquired with a shutter interval of one second, for a total of 392 images. Such an acquisition interval produces an overlap higher than the standard one. As a result, we decided to down-sample the images in correspondence of building façades and walls and to use all of them in more critical areas, such as intersections and wider areas where the buildings are located at larger distances. For this reason the processed photogrammetric block is composed of 220 images. The projection centers were determined with a bundle adjustment. Precisions were about $10 \mathrm{~cm}$, more specifically, 11 $\mathrm{cm}$ along the survey direction and the one perpendicular to it and $12 \mathrm{~cm}$ in altitude.

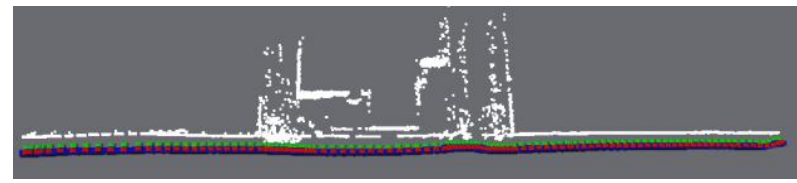

Figure 6. The photogrammetric block acquired during the survey (white points represent used tie points, while colored polygons identify camera stations)

\begin{tabular}{|c|c|}
\hline coordinate & RMS $(\mathrm{m})$ \\
\hline $\mathrm{N}$ & 0.084 \\
\hline $\mathrm{E}$ & 0.093 \\
\hline $\mathrm{h}$ & 0.096 \\
\hline
\end{tabular}

Table 7. RMS of the projection center coordinates

As a final step of our kinematic test, we performed the geometric calibration of the instrument, using the polygon and the procedure presented in paragraph 3.1.

Knowing the GPS position associated to each shot, we solved the photogrammetric problem and determined the vectors that connect each camera projection center with the GPS phase center (expressed in the camera reference system). These vectors have been determined for each camera, with a precision of $4 \mathrm{~cm}$.

For simplicity, we then decided to switch back to a single geometric vector, defined from the average projection center of the two cameras. We computed this vector for each of the image pair acquisitions performed during the survey in order to verify the georeferencing of the map as well as the quality of the block itself. On average, it was equal to $1.50 \mathrm{~m}$ with a standard deviation of $13 \mathrm{~cm}$, in agreement with the value calculated during the calibration stage.

With the aim to evaluate the accuracy of the photogrammetric solution we compared the projection center positions with the GPS ones. Those positions were interpolated with a cubic spline to the shooting time and translated to the average projection centers of the two cameras. The residuals between the two solutions (Figure 8) are no smaller than $18.6 \mathrm{~cm}$, underlining the presence of a systematic error between the two solutions: we think that this is mainly due to the fact that the calibration vector orientation with respect to the body frame (fixed to the vehicle) has been estimated with a limited accuracy. This is because the calibration vector orientation has been interpolated from estimated attitudes. Moreover, a clearly visible trend between the two solutions could be due to a drift in the photogrammetric solution, but also to some misalignment of the estimation reference frames. The two position sets are in fact converted in the cartographic national frame by applying different transformations. Nonetheless, the photogrammetric solution is in agreement with the GPS solution within the order of the required $30 \mathrm{~cm}$ tolerance for a $150 \mathrm{~m}$ path, a distance acceptable for our purposes. We think that a more accurate determination of the 3-D calibration vector could lead to a longer path. The larger residuals, after $150 \mathrm{~m}$, are also due to a degraded GPS accuracy.

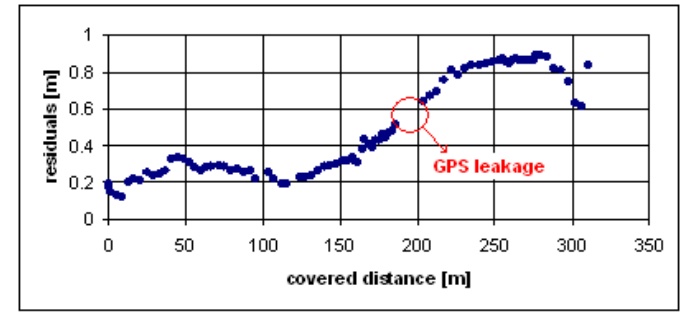

Figure 8. Differences between the photogrammetric and the GPS solutions

\section{CONCLUSIONS}

This paper presented a solution based on the implementation of different positioning systems to georeference a GPR antenna in urban areas. In those areas, due to frequent GNSS signal leakage, we cannot rely on GPS alone. A new position strategy mainly based on photogrammetry has been presented.

With the aim to ensure the greatest possible automation, we developed specific scripts for the extraction of GCPs from large scale map. The interest operator (SURF), implemented into EyeDEA, has proven successful at extracting homologous points from building façades with different lighting condition and camera angles.

However, we verified that the presence of obstacles can lead to an unfavorable configuration of the extracted tie points, especially when they are far from the cameras. 
We also studied various camera configurations. From our tests, the preferable configuration ensuring good overlap between subsequent frames and not requiring too frequent shoots was the one with two slightly converging cameras.

Because of the large number of the acquired images, we decided to down-sample them in less critical areas (e.g. in correspondence of well texture surfaces). We verified that this solution did not affect the photogrammetric block accuracies, but decreased a lot of the required computational time.

The synchronization between the two cameras and the GPS time -necessary to associate every image with its GPS positionhas been performed by software with an accuracy in the order of $0.02 \mathrm{~s}$, interpolating the GPS solution at the image acquisition time. It is preferable to increase this accuracy in order to achieve more consistency among data.

In general, the proposed method has proven reliable and the results are in agreement with the accuracy needed to georeference the GPR antenna. However, during the kinematic test, we verified that the photogrammetric solution is highly dependent on the goodness of the used GCPs.

Obviously, the map showed a variable accuracy in different town areas. There are also outliers due to map georeferencing errors or changes in urban environment. Thus, it is essential to integrate in the solution some GPS points too, that allow outlier rejection. The GPS positions can be easily acquired at the beginning and at the end of the strip or in correspondence with open spaces, such as squares or intersections.

Because the building corners are all aligned, some height point measurements (with lower precision) have been proven to allow a better constraining of the strip. We verify the proposed method accuracy comparing the photogrammetric solution with the GPS one. The differences between the two solutions are in order of the required tolerance.

The kinematic test performed is only preliminary. In the near future we are going to repeat it in order to investigate the integration of the propose method and the GPS data acquired along the trajectory, in correspondence of favorable points, such as squares and intersections.

\section{REFERENCES}

\section{References from Journals:}

Da Silva, J.F., Oliveir, P.C., Gallis, R.B.A., 2003. Development of low-cost mobile mapping system: a south American experience. 18(101), The Photogrammetric Record, pp. 5-26.

Grejner-Brzezinska, D. A., 1999. Direct Exterior Orientation of Airborne Imagery with INS/GPS system: Performance Analysis, Navigation 46(4), pp. 261-270.

Wallis, K. F., 1976. Seasonal adjustment and relation between variables. Journal of the American Statistical Association, 69 (345), pp 18-31

\section{References from Other Literature:}

Alshawa, M., Gusssenmeyer, P., Smigiel, E., 2009. Contribution of photogrammetry to the improvement of navigation data in a low cost tls based land mobile mapping system, $6^{\text {th }}$ International Symposium on Mobile Mapping.
Alshawa, M., Grussemnmeyer, P., Smigiel, E., 2009. A low cost TLS based land mobile mapping system assisted by photogrammetry, Bol. Ciênc. Geod., 15(5), pp.743-761.

Barzaghi R., Carrion D., Cazzaniga N. E., Forlani G., 2009. Vehicle Positioning in urban areas using photogrammetry and digital maps. Proceedings of the ENC-GNSSO9, Napoli (Italy).

Bay H., Ess A., Tuylelaars T., Van Gool., 2008. Speeded Robust Features (SURF). Computer Vision and Image Understanding (110), pp. 346-359.

Chaplin, B. A., Chapman, M. A., 2001. Collection of roadway asset data along an obstructed urban corridor. Proc. of the 3rd International Symposium on Mobile Mapping Technology.

Crosilla, F., Visintini, D., 1998. External Orientation of a mobile sensor via Dynamic vision of digital map points. Bollettino di Geodesia e Scienze Affini, 57(1), pp. 41-60

El-Sheimy, N., Schwarz, K. P., Gravel, M. 1995. Mobile 3-D Positioning Using GPS/INS/Video Cameras, The Mobile Mapping Symposium, Ohio State, USA, pp. 236-249.

Fischler, M., Bolles, R.,1981. Random sample consensus: a paradigm for model fitting with application to image analysis and automated cartography. Commun. Assoc. Comp. Mach., Vol 24, pp. 81-95.

Forlani, G., Roncella, R., Remondino, F., 2005. Structure and motion reconstruction of short mobile mapping image sequences. Proc. of the 7th Conf. On Optical 3D measurement techniques, Vol I, pp. 265-274, Vienna (A).

Hassan, T., Ellum, C., El-Sheimy, N., 2007. Bridging landmobile mapping using photogrammetric adjustments. ISPRS Commision Technique I. 185, pp. 66-72.

Roncella, R., Re, C., Forlani, G., 2011a. Comparison of two structure and motion strategies. ISPRS Volume XXXVIII-5/W16.

Roncella, R. Re, C., Forlani, G., 2011b. Performance evaluation of a structure and motion strategy in architecture and cultural heritage. ISPRS Volume XXXVIII-5/W16.

Roncella, R., Remondino,F., $\quad$ Forlani,G., 2005. Photogrammetric bridging of GPS outages in mobile mapping. Proc. of Videometrics VIII, SPIE-IS\&T Electronic Imaging. Vol.5665, pp. 308-319

Tao, C., Chapman, M. A., El-Sheimy, N., Chaplin, B., 1999. Towards automated processing of mobile mapping image sequences. International Archives of Photogrammetry and Remote Sensing, Vol. 32(2W1).

\section{Acknowledgements}

The authors will like to thank Riccardo Roncella for allowing the use of the software EyeDEA and for his help. We will also like to thank Ing. Nicolò Boldori who dissertated his M.Sc. degree in Environmental Engineering on these issues at Politecnico di Milano. 